\title{
A Review of Brain Stimulation Treatments for Late-Life Depression
}

\author{
Daniel M. Blumberger, MD, MSC, $F R C P C^{1,2, *}$ \\ Jonathan H. Hsu, $M D^{2}$ \\ Zafiris J. Daskalakis, MD, PhD, FRCPC ${ }^{1,2}$
}

\author{
Address \\ ${ }^{1}$ Temerty Centre for Therapuetic Brain Intervention and Campbell Family Research \\ Institute, Centre for Addiction and Mental Health, 1001 Queen St. W., Toronto, \\ ON, Canada \\ ${ }^{*}, 2$ Department of Psychiatry, University of Toronto, Toronto, 0N, Canada \\ Email: daniel.blumberger@camh.ca
}

Published online: 28 September 2015

(C) Springer International Publishing AG 2015

Keywords Late-life depression - Geriatric depression - Neuromodulation • Brain stimulation • Electroconvulsive therapy · Repetitive transcranial magnetic simulation - Transcranial direct current stimulation • Magnetic seizure therapy • Vagus nerve stimulation - Deep brain stimulation

\section{Opinion Statement}

Recurrence, relapse and resistance to first-line therapies are common and pervasive issues in the treatment of depression in older adults. As a result, brain stimulation modalities are essential treatment options in this population. The majority of data for the effectiveness of brain stimulation modalities comes from electroconvulsive therapy (ECT) studies. Current ECT trials are focused on prolonging response after a successful course and mitigating the cognitive adverse effects. Newer forms of brain stimulation have emerged; unfortunately, as with most advances in medicine older adults have not been systematically included in clinical trials. Repetitive transcranial magnetic stimulation has demonstrated efficacy in younger adults and there is emerging data to support its use in late-life depression (LLD). It will be imperative that older adults be included in future transcranial direct current stimulation and magnetic seizure therapy clinical trials. Unclear efficacy results are a concern for both vagus nerve stimulation and deep brain stimulation.

\section{Introduction}

Major depressive disorder (MDD) is the most common mental health problem in older adults [1]. Older adults with late-life depression (LLD) have higher rates of suicide compared to younger adults with depression as well as higher mortality independent of suicide $[2,3]$. The World Health Organization 
estimates that unipolar depression occurs in $7 \%$ of the general elderly population and accounts for $1.6 \%$ of total disability among those aged 60 or older [4]. This presents a significant public health concern as this age group is expected to double in the next 10 years [5].

Although psychotherapy and pharmacotherapy have been shown to be effective, a substantial proportion of older depressed adults do not respond or have early relapse with antidepressant treatment [6]. Treatmentresistant depression (TRD) is defined as failure to respond to two adequate medication trials or as a relapse during treatment [7]. The STAR*D trial showed that $60 \%$ of patients with MDD fail to respond to two antidepressant trials of optimal dose and duration, while a further $30 \%$ failed to respond to four medication trials [8].

Treatment-resistant late-life depression (TRLLD) is a common problem where up to one third of patients are affected $[9,10]$. Further, the elderly are more likely to experience relapses and recurrences than younger adults [11-13]. The failure of firstline treatment to induce remission in patients leads to impaired psychosocial function and diminished quality of life [14-16]. In addition, age-related changes that affect pharmacokinetics, pharmacodynamics, and drug interactions increase the risk of adverse effects and non-compliance with pharmacotherapy [17].

Given the challenges with pharmacotherapy and the high rates of treatment resistance in older adults, brain stimulation treatments are an important clinical consideration. Newer brain stimulation treatments have emerged as promising alternatives for TRLLD, while electroconvulsive therapy (ECT) remains an important option. ECT is the oldest, most studied and most effective treatment for TRLLD with remission rates around $60 \%[18-20]$. However, the cognitive side effects are of great concern among patients [21]. This is especially significant for geriatric populations as age is a risk factor for cognitive side effects [21]. These factors and the societal stigma towards ECT have led to the investigation of other modalities including repetitive transcranial magnetic stimulation (rTMS), transcranial direct current stimulation (tDCS), magnetic seizure therapy (MST), vagus nerve stimulation (VNS) and deep brain stimulation (DBS). The purpose of the current review is to summarize recent advances in the use of brain stimulation modalities to treat LLD.

\section{Electroconvulsive Therapy}

ECT continues to be the most established brain stimulation intervention for TRD with over 70 years of data [22]. Despite the paucity of clinical trials specifically focused on LLD as identified by a Cochrane review [23], the majority of studies have included older adults. These studies demonstrate that ECT is one of the most effective treatments for MDD with response around $60 \%$ [18]. Recent studies in ECT for LLD focus on speed of remission in non-TRD patients, stimulus pulse width and maintenance protocols to address issues of neurocognitive side effects and relapse rates.

A very recent study [24•] reanalyzed subjects aged over 60 from two double blind, controlled trials to compare the speed of remission using ECT versus medication in elderly with MDD but not necessarily with TRD. The first selected trial [25] recruited adult MDD patients and randomized them to twice weekly right unilateral (RUL) brief pulse $(1.0 \mathrm{msec})$ or ultra-brief pulse (0.3-0.37 msec) ECT eight times seizure threshold. The second trial [26] randomized elderly patients to venlafaxine or nortriptyline. The comparative study found that mean time to remission was 3.1 weeks (SD 1.1) for the ECT group and 4.0 weeks (SD 1.0) for the medication group. The adjusted hazard ratio for remission within 5 weeks (ECT v. medication) was 3.4 (95\% CI 1.9-6.2). These findings substantiate the clinical impression that ECT has an important role in patients with severe LLD.

The speed of remission in LLD was supported by preliminary results from an ongoing trial [27] (ClinicalTrials.gov Identifier: NCT01028508). The Prolonging Remission in Depressed Elderly (PRIDE) study reported a mean of 7.3 (SD 3.1) ECT sessions to reach remission, which is a comparable duration of approximately 2.5 weeks with ECT administration three times weekly. This study has recruited 237 patients with unipolar depression over 60 years of age in phase 1 to receive ultra-brief pulse RUL ECT augmented with venlafaxine. The study will randomize patients in phase 2 to venlafaxine plus lithium or venlafaxine plus lithium with flexible maintenance ECT. The study design in older adults is informed by earlier trials in that they have included both younger and older adults that suggest enhanced efficacy of ECT with concomitant use of a noradrenergic antidepressant [20]. In this ECT and medication augmentation study, high dosage RUL, standard pulse width, ECT was also shown to be equally as effective as moderate dosage bitemporal ECT with reduced cognitive adverse effects. In the 6-month continuation 
phase of this study, however, a $50 \%$ relapse rate was observed despite continuation pharmacotherapy with

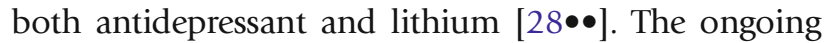
PRIDE trial will help elucidate whether flexible maintenance ECT may reduce relapse rates in older adults. In addition, the cognitive adverse effect data in this large sample will inform decisions around when to select RUL-UB ECT compared to other emerging brain stimulation modalities.

\section{Repetitive Transcranial Magnetic Simulation}

Repetitive transcranial magnetic simulation (rTMS) is a brain stimulation method that uses magnetic field pulses, rather than an electrical current, and does not induce a seizure. The procedure requires a stimulator and coil to produce an electromagnetic field. A typical treatment course is 5 days per week between 4 and 6 weeks. In general, the treatment has a favourable adverse effect profile with common side effects including scalp discomfort and transient headache. There are no cognitive adverse effects reported with rTMS. In 2008, the FDA approved rTMS as a treatment for depression for patients not responding to at least one antidepressant medication with a maximum age of 69 [29].

Despite the relatively large number of rTMS studies completed to date, there is a paucity of studies evaluating the efficacy of rTMS in TRLLD specifically. A number of older reports have suggested that older age is a negative predictor of response to rTMS [30-34]. However, these studies used suboptimal stimulation parameters particularly with respect to the stimulation intensity needed to overcome the prefrontal atrophy that occurs with advancing age. The proposed mechanism for these negative findings is likely related to the increased scalpto-cortex distance in the elderly [30]. Imaging studies [34, 35] and a small uncontrolled clinical pilot study [36] have suggested a correlation between antidepressant effect of rTMS and scalp-to-cortex distance. Nahas et al. (2004) used an open design in which they adjusted stimulus intensity based on the distance of the scalp to the cortex and used MRI co-registration to target the dorsolateral prefrontal cortex (DLPFC), in 18 older subjects [36]. The average intensity required was $114 \%$, significantly higher than the intensity used in other treatment trials at the time. Interestingly, a more recent randomized controlled trial in an elderly sample with depression and cerebrovascular damage found unilateral rTMS at $110 \%$ stimulation intensity resulted in a significant, but modest, $27.3 \%$ remission rate [37].
The rTMS field has moved to using $120 \%$ of the motor threshold intensity across the age range of subjects and as a result meta-analyses of more recent DLPFC rTMS have not found that older age is a negative predictor of response [38, 39]. Some preliminary data suggests that older adults may respond better to a sequential bilateral form of rTMS where low frequency (i.e. $1 \mathrm{~Hz}$ ) right-sided stimulation is immediately followed by high-frequency (i.e. $10 \mathrm{~Hz}$ ) left-sided stimulation, using $120 \%$ stimulation intensity [40•]. Other preliminary data suggests that older adults may respond better with a coil that has a larger induced electrical field than standard figure of eight coils [41].

Further study of rTMS in older adults is warranted given that optimal treatment parameters have demonstrated more promising results that earlier studies. Controlled studies across the age spectrum of older adults (i.e. over age 70) are needed to confirm efficacy and tolerability in this sub-group of older adults. Older adults may be particularly able to benefit from rTMS as daily schedules may be more conducive to the 5 days a week treatment schedule. rTMS has good patient acceptability due to the favourable adverse effect profile, in particular the lack of cognitive side effects.

\section{Magnetic Seizure Therapy}

As a modality that has similarities to TMS and ECT, MST induces a seizure by utilizing a train of high-intensity repetitive magnetic field pulses that induce an electric field within the brain. The procedure for MST is the same as for ECT whereby patients receive low-dose anaesthesia and paralytic agent prior to the induction of the seizure. Magnetic field pulses are far more focal as they are not impeded or shunted by the skull or cerebrospinal fluid, thus preventing diffuse brain activation [42]. As a result there are far fewer cognitive side effects associated with MST compared with ECT [43-46].

The largest published, randomized controlled trial [47] to date $(n=20)$ compared MST (twin coil, $100 \mathrm{~Hz}$, up to 600 pulses) to ECT (RUL, 0.5 brief pulse wave form) in TRD patients. The results showed statistically significant and similar antidepressant response in both groups. No significant cognitive side effects were noted in either group; however, those who had MST showed earlier time to recovery and reorientation post procedure. The study age inclusion restrictions were 18-65, which limits interpretations specific for LLD.

More recent pilot data continue to investigate the clinical effects and safety of $100 \mathrm{~Hz}$ MST [48, 49]. The cohort of younger TRD patients $(n=13$, mean age 46.8 , 
SD 14.8) received $100 \mathrm{~Hz}$ MST in trains up to $10 \mathrm{~s}$ for up to 18 treatments. There were significant group reductions in depression scores and at study conclusion, five were responders $(38.5 \%)$. There were no cognitive side effects.

Recent MST studies have failed to include geriatric patients, which prevents conclusions specific to LLD. A recent, case report described the induction of mania in a geriatric patient severe bipolar depression with psychotic features in the context of an open label MST trial that includes older adults up to age 85 [50]. Though the induction of mania was a serious adverse event, switches into mania with ECT also occur [51, 52]. The promising cognitive safety profile of MST may make it a more appealing approach for older adults with TRLLD. Until a definitive study comparing MST to ECT is conducted, questions will remain surrounding the relative efficacy balanced against the more advantageous cognitive adverse effect profile. Clinicians will demand to know if there is any efficacy lost with MST compared to ECT to gain the better cognitive adverse effect profile. Given that ECT is often indicated in older adults with severe depression, future MST studies must include this population.

\section{Transcranial Direct Current Stimulation}

tDCS is a non-invasive neuromodulation modality that involves the placement of two surface scalp electrodes and the application of a weak, non-convulsive current (1-2 mA) to the brain. This mode of stimulation has been shown to effect cortical areas by modulating cerebral flood flow, metabolism and brain-derived neurotrophic factors [53-55]. Initial studies [56] in the 1960s produced mixed results as a result of methodological differences and confounding. There has been a renewed interest in the last decade with several trials and metaanalyses in the last few years, in particular.

A meta-analysis [57] in 2012 included 10 studies of which 6 were randomized controlled trials [58-63]. Pooled results $(n=176)$ were reported as a continuous outcome showing that active tDCS was more effective in reducing depressive symptoms than sham tDCS. However, a meta-analysis in 2013 which included an additional trial [64] noted no differences between active and sham tDCS in reporting a categorical outcome in the form of remission or response rates in the pooled results $(n=200)$.

Yet another meta-analysis in 2014 added to previous results by including an additional recent large trial [65]. This trial compared tDCS versus sertraline in a $2 \times 2$ factorial design that included sertraline/placebo and active/sham tDCS (anodal left/cathodal right prefrontal) in 120 moderate to severe MDD. tDCS only was superior to placebo/sham tDCS. The combined treatment group (sertraline/active tDCS) also had greater therapeutic benefit compared to sertraline only. The tDCS only versus sertraline group had similar treatment responses. In this trial, inclusion criteria were limited those aged 18-65.

The meta-analysis in 2014 included both categorical and continuous outcomes in treatment response. With the additional larger study, the pooled results $(n=259)$ showed that active tDCS was significantly superior to sham in symptom score, response and remission rates. Notably, it did not include the sertraline/active tDCS group from the new trial (which had the greatest treatment response) as it differed from the active treatment group in all other trials. The mean age of the population was 43.62 years (SD 10) owing to the exclusion of older patients in several trials. A meta-regression of the influence of age on the outcome did not show any correlation.

There is increasing evidence that supports the therapeutic benefit of tDCS in MDD. Further work is needed to elucidate the optimal parameters for neuromodulation and the inclusion of adequate samples of older populations to adequately investigate its utility in LDD. Data from the use of tDCS to enhance motor recovery in older stroke patients [66] suggests that older adults with depression may also stand to benefit from this intervention.

\section{Vagus Nerve Stimulation}

Vagus nerve stimulation (VNS) is an invasive treatment that involves the implantation of an electrode around the vagus nerve that delivers intermittent electrical signals to the brain. VNS was initially approved by the FDA for treatment of refractory epilepsy in 1997 [29]. It was investigated for its antidepressant effects after improvement in mood in treated patients and in 2005, it was approved as a therapy for TRD by the FDA. Indications include failure of four other treatments, severe and recurrent MDD lasting 2 years or more. A recent industry funded meta-analysis concluded that response and remission rates with VNS were better than what could be with treatment as usual [67]. All studies reviewed were industry sponsored, including unpublished, patientlevel data, which were mostly observational in nature and lacked a placebo control. It is difficult to make any conclusions regarding the effectiveness of VNS in older 
adults with depression as the six included trials had strict inclusion criteria and an overall mean age of 47.8 (SD 10.2).

Conversely, an independent systematic review concluded that there was insufficient evidence to evaluate VNS as a treatment for TRD. The study identified only one randomized controlled trial [68] that covered depression ( $n=235)$, which reported no statistically significant differences between the active intervention and placebo group (mean age 46.5, SD 9.0). The review identified that positive results were limited to uncontrolled trials.

There is a scarcity of data regarding VNS in the elderly depressed; however, there is a suggestion that VNS is safe and reasonably well tolerated by older adults from several small studies for indications other than depression $[69,70]$. Overall, there is insufficient safety and efficacy data to guide practitioners on the use of VNS for TRLLD.

\section{Deep Brain Stimulation}

DBS is the most invasive of the brain stimulation modalities. It involves neurosurgical implantation of electrodes in the brain to target neuroanatomical areas with a generator implanted in the chest. It has been approved by the FDA for Parkinson's and obsessivecompulsive disorder, but not TRD. Imaging studies which have localized the Brodmann area 25 and subgenual cingulate cortex in TRD have generated interest in DBS for depression [71].
Early studies of DBS in TRD have been limited to small case series [72]. Results show that approximately half of patients responded. In younger study subjects, one study [73] included 10 MDD patients and 7 bipolar patients (mean age 42.0, SD 8.9) who had failed at least 4 adequate antidepressant trials and employed a 4-week sham lead-in phase and active stimulation for 24 weeks. Despite a significant placebo effect in the sham lead-in phase, there was a sustained decrease in depression and increase in function was observed (41\% responded at 24 weeks, $36 \%$ after 1 year, $92 \%$ after 2 years). More recent studies have begun to investigate the relationship between stimulus parameters and clinical efficacy. A small double blind pilot study [74] ( $n=4)$ randomly changed frequency and pulse widths weekly in TRD patients who underwent DBS (aged 46-56). They found that longer pulse widths (270-450 $\mu$ s) were associated with more robust antidepressant effects.

Concerns around efficacy of DBS compared to placebo have begun to emerge as trials emerge in the literature [75]. Other targets for DBS such as the medial forebrain bundle have shown significant promise and remain an option for future DBS studies [76]. The neurobiological underpinnings of depression in older adults may be more complex, and work to identify appropriate neuroanatomical targets is necessary before moving forward with clinical trials in LLD.

\section{Conclusion}

The emergence of newer brain stimulation treatments is a necessary evolution in the treatment of depression as progress in psychotherapy and pharmacotherapy has stalled advances in the oldest form brain stimulation for LLD; ECT involves refinement in electrode placement using unilateral stimulation with ultra-brief pulse widths in an attempt to reduce cognitive adverse effects. However, cognitive adverse effects are not eliminated and public perception of the treatment remains an obstacle for patient acceptance. The results of the PRIDE study (ClinicalTrials.gov Identifier: NCT01028508) should further clarify the cognitive adverse effects of ECT in older adults with ultra-brief pulse width ECT. rTMS has emerged as an alternative treatment option for younger patients with resistant depression. Data suggests that adults up to age 69 can also benefit from this treatment. A bilateral treatment approach may be another consideration when treating older adults with rTMS. More studies of older adults above the age of 70 are necessary to clarify the role of rTMS in LLD in this population. tDCS may have a role in less severe and resistant forms of 
depression, yet efficacy data in older adults is lacking. The low cost and potential portability of this treatment make it an attractive alternative should efficacy data support its use. MST is still under investigation as a cognitively neutral alternative to ECT. Since older adults seem to show robust response to electrically induced seizures, they may also show similar effects with electromagnetically induced seizures. The favourable cognitive profile of MST and the lack of stigma may lead to enhanced patient acceptability and as a result a larger proportion of patients treated who would otherwise refuse an effective treatment option. Future clinical trials comparing the efficacy and cognitive adverse effects of MST to ECT should include older adults. Very few older adults have been included in VNS and DBS clinical trials and thus the role of these treatments in LLD is unknown.

\section{Compliance with Ethics Guidelines}

\section{Conflict of Interest}

Daniel M. Blumberger receives research support from that Canadian Institutes of Health Research (CIHR), National Institute of Mental Health (NIMH), Temerty Family through the CAMH Foundation and the Campbell Research Institute and in-kind equipment support for an investigator-initiated study from MagVenture/Tonika and research and in-kind equipment support for an investigator-initiated study from Brainsway Ltd.

Jonathan H. Hsu declares no conflict of interest.

Zafiris J. Daskalakis received external funding through Neuronetics and Brainsway Ltd., Aspect Medical, travel allowance through Pfizer and Merck, speaker funding through Sepracor Inc. and AstraZeneca. He has served on the advisory board for Hoffmann-La Roche Limited, and received funding from the Brain and Behaviour Research Foundation, CIHR, Ontario Mental Health Foundation, and the Temerty Family and Grant Family and through the Centre for Addiction and Mental Health (CAMH) Foundation and the Campbell Research Institute.

\section{Human and Animal Rights and Informed Consent}

This article does not contain any studies with human or animal subjects performed by any of the authors.

\section{References and Recommended Reading}

Papers of particular interest, published recently, have been highlighted as:

- Of importance

•• Of major importance

1. Husain MM, Rush AJ, Sackeim HA, et al. Age-related characteristics of depression: a preliminary STAR*D Report. Am J Geriatr Psychiatr. 2005;13(10):852-60.

2. Bruce ML, Ten Have TR, Reynolds 3rd CF, et al. Reducing suicidal ideation and depressive symptoms in depressed older primary care patients: a randomized controlled trial. JAMA. 2004;291(9):1081-91.
3. Turvey CL, Conwell $\mathrm{Y}$, Jones MP, et al. Risk factors for late-life suicide: a prospective, community-based study. Am J Geriatr Psychiatr. 2002;10(4):398-406.

4. World Health Organization. Mental health and older adults. 2013.

5. Belanger A, Martel L. Caron-Malenfant r, Statistics Canada. Demography Division. Population 
projections for Canada, provinces and territories, 20052031. Ottawa: Statistics Canada, Demography Division; 2005.

6. Mulsant BH, Pollock BG. Treatment-resistant depression in late life. J Geriatr Psychiatry Neurol. 1998;11(4):186-93.

7. Thase M, Rush A. Treatment resistant depression. New York: Raven Press Ltd.; 1995.

8. Rush JA, Trivedi MH, Wisniewski SR, et al. Acute and longer-term outcomes in depressed outpatients requiring one or several treatment steps: a STAR* D report. Am J Psychiatry. 2006;163(11):1905-17.

9. Fava M, Davidson KG. Definition and epidemiology of treatment-resistant depression. Psychiatr Clin N Am. 1996;19(2):179-200.

10. Baldwin RC. Refractory depression in late life: a review of treatment options. Rev Clin Gerontol.

1996;6(4):343-8.

11. Mulsant BH, Houck PR, Gildengers AG, et al. What is the optimal duration of a short-term antidepressant trial when treating geriatric depression? J Clin Psychopharmacol.

2006;26(2):113-20.

12. Reynolds 3rd CF, Dew MA, Pollock BG, et al. Maintenance treatment of major depression in old age. $\mathrm{N}$ Engl J Med. 2006;354(11):1130-8.

13. Tew JD, Mulsant BH, Houck PR, et al. Impact of prior treatment exposure on response to antidepressant treatment in late life. Am J Geriatr Psychiatr. 2006;14(11):957-65.

14. Blazer DG. The prevalence of depressive symptoms. J Gerontol A Biol Sci Med Sci. 2002;57(3):M150-1.

15. Keller MB. Past, present, and future directions for defining optimal treatment outcome in depression: remission and beyond. JAMA. 2003;289(23):3152-60.

16. Doraiswamy PM, Khan ZM, Donahue RMJ, Richard NE. Quality of life in geriatric depression - a comparison of remitters, partial responders, and nonresponders. Am J Geriatr Psychiatr. 2001;9(4):423-8.

17. Lotrich FE, Pollock BG. Aging and clinical pharmacology: implications for antidepressants. J Clin Pharmacol. 2005;45(10):1106-22.

18. Lisanby SH. Electroconvulsive therapy for depression. N Engl J Med. 2007;357(19):1939-45.

19. Kellner CH, Knapp R, Husain MM, et al. Bifrontal, bitemporal and right unilateral electrode placement in ECT: randomised trial. Br J Psychiatry.

2010;196(3):226-34.

20. Sackeim HA, Dillingham EM, Prudic J, et al. Effect of concomitant pharmacotherapy on electroconvulsive therapy outcomes: short-term efficacy and adverse effects. Arch Gen Psychiatry. 2009;66(7):729-37.

21. Sackeim HA, Prudic J, Fuller R, et al. The cognitive effects of electroconvulsive therapy in community settings. Neuropsychopharmacology. 2006;32(1):24454.

22. American Psychiatric Association Committee on Electroconvulsive T. The Practice of Electroconvulsive Therapy: Recommendations for Treatment, Training, and Privileging. Washington, DC: American Psychiatric Association. 2001.

23. Stek M, Hoogendijk W, Beekman A. Electroconvulsive therapy for the depressed elderly. The Cochrane Library 2003

24. Spaans H-P, Sienaert P, Bouckaert F, et al. Speed of remission in elderly patients with depression: electroconvulsive therapy v. medication. Br J Psychiatry. 2015;206(1):67-71.

This is an important secondary analysis demonstrating the superiority in speed of remission with ECT compared to pharmacotherapy.

25. Spaans H-P, Verwijk E, Comijs HC, et al. Efficacy and cognitive side effects after brief pulse and ultrabrief pulse right unilateral electroconvulsive therapy for major depression: a randomized, double-blind, controlled study. J Clin Psychiatr. 2013;74(11):e1029-36.

26. Kok R, Nolen W, Heeren T. Venlafaxine versus nortriptyline in the treatment of elderly depressed inpatients: a randomised, double-blind, controlled trial. Int J Geriatr Psychiatr. 2007;22(12):1247-54.

27. Kellner CH, Geduldig ET, Knapp RG, et al. More data on speed of remission with ECT in geriatric depression. Br J Psychiatry. 2015;206(2):167.

28.• Prudic J, Haskett RF, McCall WV, et al. Pharmacological strategies in the prevention of relapse after electroconvulsive therapy. J ECT. 2013;29(1):3-12.

Despite the high remission with ECT this study showed high relapse rates with aggressive augmentation pharmacotherapy with lithium.

29. Manepalli J, Sapkota N. Neuromodulation therapies in the elderly depressed patient. Curr Geriatr Rep. 2014;3(4):229-36.

30. Figiel GS, Epstein C, McDonald WM, et al. The use of rapid-rate transcranial magnetic stimulation (rTMS) in refractory depressed patients. J Neuropsychiatry Clin Neurosci. 1998;10(1):20-5.

31. Fregni F, Marcolin MA, Myczkowski M, et al. Predictors of antidepressant response in clinical trials of transcranial magnetic stimulation. Int J

Neuropsychopharmacol. 2006;9(6):641-54.

32. Manes $\mathrm{F}$, Jorge $\mathrm{R}$, Morcuende $\mathrm{M}$, et al. A controlled study of repetitive transcranial magnetic stimulation as a treatment of depression in the elderly. Int Psychogeriatr. 2001;13(2):225-31.

33. Mosimann UP, Marre SC, Werlen S, et al. Antidepressant effects of repetitive transcranial magnetic stimulation in the elderly: correlation between effect size and coil-cortex distance. Arch Gen Psychiatry. 2002;59(6):560-1.

34. Mosimann UP, Schmitt W, Greenberg BD, et al. Repetitive transcranial magnetic stimulation: a putative add-on treatment for major depression in elderly patients. Psychiatry Res. 2004;126(2):123-33.

35. Kozel FA, Nahas Z, deBrux C. How coil-cortex distance relates to age, motor threshold, and antidepressant response to repetitive transcranial magnetic stimulation. J Neuropsychiatry Clin Neurosci. 2000;12(3):376-84. 
36. Nahas Z, Li X, Kozel FA, et al. Safety and benefits of distance-adjusted prefrontal transcranial magnetic stimulation in depressed patients 55-75 years of age: a pilot study. Depress Anxiety. 2004;19(4):249-56.

37. Jorge RE, Moser DJ, Acion L, Robinson RG. Treatment of vascular depression using repetitive transcranial magnetic stimulation. Arch Gen Psychiatry. 2008;65(3):268-76.

38. Berlim MT, van den Eynde F, Tovar-Perdomo S, Daskalakis ZJ. Response, remission and drop-out rates following high-frequency repetitive transcranial magnetic stimulation (rTMS) for treating major depression: a systematic review and meta-analysis of randomized, double-blind and sham-controlled trials. Psychol Med. 2014;44(2):225-39.

39. Gross M, Nakamura L, Pascual-Leone A, Fregni F. Has repetitive transcranial magnetic stimulation (rTMS) treatment for depression improved? A systematic review and meta-analysis comparing the recent vs. the earlier rTMS studies. Acta Psychiatr Scand. 2007;116(3):165-73.

40. Blumberger DM, Mulsant BH, Fitzgerald PB, et al. A randomized double-blind sham-controlled comparison of unilateral and bilateral repetitive transcranial magnetic stimulation for treatment-resistant major depression. World J Biol Psychiatry. 2012;13(6):423-35. Adults over age 60 were included in this study and seem to respond better with sequential bilateral rTMS using $120 \%$ of the motor threshold.

41. Levkovitz Y, Harel EV, Roth Y, et al. Deep transcranial magnetic stimulation over the prefrontal cortex: evaluation of antidepressant and cognitive effects in depressive patients. Brain Stimul. 2009;2(4):188-200.

42. Deng Z-D, Lisanby SH, Peterchev AV. Electric field strength and focality in electroconvulsive therapy and magnetic seizure therapy: a finite element simulation study. J Neural Eng. 2011;8(1):016007.

43. Lisanby SH. Update on magnetic seizure therapy: a novel form of convulsive therapy. J ECT.

2002;18(4):182-8.

44. Luber B, Nobler MS, Moeller JR, et al. Quantitative EEG during seizures induced by electroconvulsive therapy: relations to treatment modality and clinical features. II. Topographic analyses. J ECT. 2000;16(3):229-43.

45. Nobler MS, Oquendo MA, Kegeles LS, et al. Decreased regional brain metabolism after ECT. Am J Psychiatr. 2001;158(2):305-8.

46. McClintock SM, Tirmizi O, Chansard M, Husain MM. A systematic review of the neurocognitive effects of magnetic seizure therapy. Int Rev Psychiatr. 2011;23(5):413-23.

47. Kayser S, Bewernick BH, Grubert C, et al. Antidepressant effects, of magnetic seizure therapy and electroconvulsive therapy, in treatment-resistant depression. J Psychiatr Res. 2011;45(5):569-76.

48. Fitzgerald PB, Hoy KE, Herring SE, et al. Pilot study of the clinical and cognitive effects of high-frequency magnetic seizure therapy in major depressive disorder. Depression Anxiety. 2013;30(2):129-36.
49. Hoy KE, Thomson RH, Cherk M, et al. Effect of magnetic seizure therapy on regional brain glucose metabolism in major depression. Psychiatry Res.

2013;211(2):169-75.

50. Noda Y, Daskalakis ZJ, Fitzgerald PB, et al. Magnetic seizure therapy-induced mania: a report of 2 cases. J ECT. 2015;31(1):e4-6.

51. Devanand DP, Sackeim HA, Decina P, Prudic J. The development of mania and organic euphoria during ECT. J Clin Psychiatry. 1988;49(2):69-71.

52. Lee J, Arcand L, Narang P, Lippmann S. ECT-induced mania. Innov Clin Neurosci. 2014;11(11-12):27-9.

53. Fritsch B, Reis J, Martinowich K, et al. Direct current stimulation promotes BDNF-dependent synaptic plasticity: potential implications for motor learning. Neuron. 2010;66(2):198-204.

54. Nitsche MA, Paulus W. Excitability changes induced in the human motor cortex by weak transcranial direct current stimulation. J Physiol. 2000;527(Pt 3):633-9.

55. Zheng X, Alsop DC, Schlaug G. Effects of transcranial direct current stimulation (tDCS) on human regional cerebral blood flow. Neuroimage. 2011;58(1):26-33.

56. Costain R, Redfearn JW, Lippold OC. A controlled trial of the therapeutic effect of polarization of the brain in depressive illness. Br J Psychiatr J Ment Sci.

1964;110:786-99.

57. Kalu UG, Sexton CE, Loo CK, Ebmeier KP. Transcranial direct current stimulation in the treatment of major depression: a meta-analysis. Psychological medicine 2012:1-10

58. Boggio PS, Rigonatti SP, Ribeiro RB, et al. A randomized, double-blind clinical trial on the efficacy of cortical direct current stimulation for the treatment of major depression. Int J Neuropsychopharmacol Off Sci Journal Coll Int Neuropsychopharmacologicum. 2008;11(2):249-54.

59. Fregni F, Boggio PS, Nitsche MA, et al. Treatment of major depression with transcranial direct current stimulation. Bipolar Disord. 2006;8(2):203-4.

60. Fregni F, Boggio PS, Nitsche MA, et al. Cognitive effects of repeated sessions of transcranial direct current stimulation in patients with depression. Depress Anxiety. 2006;23(8):482-4.

61. Loo CK, Alonzo A, Martin D, et al. Transcranial direct current stimulation for depression: 3-week, randomised, sham-controlled trial. Br J Psychiatr J Ment Sci. 2012;200(1):52-9.

62. Loo CK, Sachdev P, Martin D, et al. A double-blind, sham-controlled trial of transcranial direct current stimulation for the treatment of depression. Int J Neuropsychopharmacol Off Sci J Coll Int Neuropsychopharmacologicum. 2010;13(1):61-9.

63. Palm U, Schiller C, Fintescu Z, et al. Transcranial direct current stimulation in treatment resistant depression: a randomized double-blind, placebo-controlled study. Brain Stimul. 2012;5(3):242-51.

64. Blumberger DM, Tran LC, Fitzgerald PB, et al. A randomized double-blind sham-controlled study of transcranial direct current stimulation for treatment- 
resistant major depression. Front Psychiatr Front Res Found. 2012;3:74.

65. Brunoni AR, Valiengo L, Baccaro A et al. . The Sertraline vs Electrical Current Therapy for Treating Depression Clinical Study: Results From a Factorial, Randomized, Controlled Trial. JAMA psychiatry 2013:1-9

66. Marquez J, van Vliet P, McElduff $P$, et al. Transcranial direct current stimulation (tDCS): does it have merit in stroke rehabilitation? A systematic review. Int J Stroke. 2015;10(3):306-16.

67. Berry SM, Broglio K, Bunker M, et al. A patient-level meta-analysis of studies evaluating vagus nerve stimulation therapy for treatment-resistant depression. Med Devices. 2013;6:17-35.

68. Rush AJ, Marangell LB, Sackeim HA, et al. Vagus nerve stimulation for treatment-resistant depression: a randomized, controlled acute phase trial. Biol Psychiatry. 2005;58(5):347-54.

69. Sirven JI, Sperling M, Naritoku D, et al. Vagus nerve stimulation therapy for epilepsy in older adults. Neurology. 2000;54(5):1179-82.

70. Merrill CA, Jonsson MA, Minthon L, et al. Vagus nerve stimulation in patients with Alzheimer's disease: additional follow-up results of a pilot study through 1 year. J Clin Psychiatry. 2006;67(8):1171-8.
71. Mayberg HS, Lozano AM, Voon V, et al. Deep brain stimulation for treatment-resistant depression. Neuron. 2005;45(5):651-60.

72. Lakhan SE, Callaway E. Deep brain stimulation for obsessive-compulsive disorder and treatment-resistant depression: systematic review. BMC Res Notes. 2010;3:60.

73. Holtzheimer PE, Kelley ME, Gross RE, et al. Subcallosal cingulate deep brain stimulation for treatmentresistant unipolar and bipolar depression. Arch Gen Psychiatry. 2012;69(2):150-8.

74. Ramasubbu R, Anderson S, Haffenden A, et al. Doubleblind optimization of subcallosal cingulate deep brain stimulation for treatment-resistant depression: a pilot study. J Psychiatry Neurosci. 2013;38(5):325-32.

75. Dougherty DD RA, Carpenter LL, Howland RH, Bhati MT, O'Reardon JP, Eskandar EN, et al. A randomized sham-controlled trial of deep brain stimulation of the ventral capsule/ventral striatum for chronic treatmentresistant depression. Biol Psychiatry. 2014. doi:10. 1016/j.biopsych.2014.11.023.

76. Galvez JF, Keser Z, Mwangi B, et al. The medial forebrain bundle as a deep brain stimulation target for treatment resistant depression: A review of published data. Prog Neuropsychopharmacol Biol Psychiatry. 2015;58:59-70. 